\title{
SOLUTION OF THE DIFFERENTIAL EQUATIONS OF MOTION OF A PROJECTILE IN A MEDIUM OF QUASI-NEWTONIAN RESISTANCE*
}

\author{
BY \\ HARRY POLACHEK \\ Naval Ordnance Laboratory, White Oak, Md.
}

1. Introduction. An analytic solution for the basic differential equations of exterior ballistics has never been found. These equations express the path of a projectile in a retarding medium such as air. In practice these equations are solved by a method of successive approximations for each new set of constants or initial conditions. This is a very laborious process.

The principal difficulty is the complexity of the law of air resistance. Several solutions have been found on the basis of simple retardation laws. These, however, are not the true laws of resistance encountered by a projectile, as shown by experiment. The only solution which is of any practical value to ballisticians is that found by John Bernoulli for the Newtonian law of air resistance, $R=K V^{2}$. This is used in many cases where there are no accurate experimental data available.

Although resistance encountered by a projectile never behaves exactly according to Newton's law, there are many instances where the deviation from this law is "small". This is true for projectiles moving with velocities less than that of sound. In this paper a solution is obtained for the differential equations of motion of a body in a medium in which the resistance law is almost Newtonian, or quasi-Newtonian. This is done by making use of the theory of differential variations which takes into account differentials of the first order only, neglecting differentials of higher order.

In an illustrative example in the text the author works out a trajectory of a shell with an initial velocity of $300 \mathrm{~m} / \mathrm{s}$ (the velocity of sound is approximately $340 \mathrm{~m} / \mathrm{s}$ ) and an angle of elevation of $60^{\circ}$, by both the new method and the standard method of successive approximations. The ranges obtained by the two methods are identical to the nearest meter, each giving a range of 4240 meters. Since the initial velocity in the illustrative problem is almost that of sound and the angle of elevation is rather large it may be expected that this method will give accurate results for all trajectories with initial velocities below sound. It should be pointed out that the variation of density with altitude is also taken into account.

The derivation of explicit expressions is confined in this paper to the Newtonian law of resistance because of its practical usefulness. However, it is apparent from subsequent discussions that the methods used are quite general, and are applicable with slight modifications to any resistance law for which a solution to the equations of motion can be obtained in finite form.

2. Resistance of a moving projectile. If a body is moving in a medium other than a vacuum, it is retarded according to some complex law which is related to the density of the medium, the size and shape of the moving body, and its velocity. An exact law which governs the amount of retardation, or deceleration has not yet been found. The motion is influenced by a number of factors which are too difficult for a complete theoretical analysis. For instance, when a projectile moving in air exceeds the velocity

${ }^{*}$ Received Feb. 14, 1948. 
of sound, it produces a pattern of shock waves which use up its energy and greatly reduce its velocity. Experimental curves are now available which give the retardation for several shapes of projectiles and for varying velocities. From these curves it is apparent that the velocity of sound acts as a dividing line, at which the curve invariably experiences a steep and almost discontinuous rise due to the shock waves propagated by the moving body. The subsonic region is a region of quasi-Newtonian resistance to which the methods of this paper are applicable. In this region the law of resistance governing the moving body deviates from Newton's quadratic law of resistance, $R=$ $K V^{2}$, by a "small" amount.

3. The equations of motion. For simplicity we will consider a projectile moving in a vertical plane, acted upon by the force of gravity $m g$ (where $m$ is the mass of the projectile) in a downward direction, and by a retardation force $m R$ in a direction opposite to that of its motion. Let us fix a set of axes in this plane, $y$ being the vertical axis and $x$ the horizontal axis. The differential equations of motion may then be written in the form

$$
\begin{aligned}
& \frac{d^{2} x}{d t^{2}}=-E \frac{d x}{d t} \\
& \frac{d^{2} y}{d t^{2}}=-E \frac{d y}{d t}-g
\end{aligned}
$$

where $E=R / V$. In the general ballistic problem $E$ is a function of $y$, the altitude, as well as of $V$, the velocity. $E$ is usually written equal to $K G(V) H(y)$, where $K$ is a constant characteristic of the projectile, $G(V)$ is a function of the velocity alone, and $H(y)$ is the density of the air aloft and is a function of $y$ alone.

4. Solutions of Bernoulli and D'Alembert. ${ }^{1}$ In 1719 John Bernoulli had obtained the solution of the above set of differential equations on the simplifying assumption that $R=K V^{n}$. Later, in 1744 d'Alembert integrated the same equations for the slightly more general resistance law $R=a+K V^{n}$. We are particularly interested in the special case $R=K V^{2}$, but since it does not present any additional difficulties we shall repeat here the derivation of the more general solution due to d'Alembert. In Cartesian coordinates $d x / d t=V \cos \theta$ and $d y / d t=V \sin \theta$, where $\theta$ is the angle between the tangent at any point of the trajectory and the $x$-axis. Equations (1) may then be written

$$
\begin{aligned}
& \frac{d}{d t}(V \cos \theta)=-\left(a+K V^{n}\right) \cos \theta \\
& \frac{d}{d t}(V \sin \theta)=-\left(a+K V^{n}\right) \sin \theta-g .
\end{aligned}
$$

Differentiating the left-hand side of Eqs. (2), then multiplying by $\sin \theta$ and $\cos \theta$, respectively, and subtracting, we obtain

$$
\frac{d t}{d \theta}=-\frac{V}{g \cos \theta} .
$$

'K. J. Cranz and K. Becker, Exterior ballistics (Vol. I of Handbook of Ballistics), His Majesty's Stationery Off., London, 1921, Ch. IV. 
It follows immediately that

$$
\begin{aligned}
& \frac{d x}{d \theta}=\frac{d x}{d t} \frac{d t}{d \theta}=-\frac{V^{2}}{g} \\
& \frac{d y}{d \theta}=\frac{d y}{d t} \frac{d t}{d \theta}=-\frac{V^{2}}{g} \tan \theta \\
& \frac{d s}{d \theta}=\frac{d s}{d t} \frac{d t}{d \theta}=-\frac{V^{2}}{g \cos \theta} .
\end{aligned}
$$

Substituting Eq. (3) into Eq. (1) and expanding the left-hand side, we also obtain the equation

$$
g \cos \theta\left(V^{-n-1}\right) d V-(a+g \sin \theta) V^{-n} d \theta=K d \theta .
$$

This equation may readily be integrated, giving an expression for $V$ in terms of $\theta$,

$$
\begin{aligned}
V^{-n}=\cos ^{n} \theta & \exp \left[-n g^{-1} \int a(\cos \theta)^{-1} d \theta\right] \\
\times & \left\{-\frac{n}{g} \int \frac{K \exp \left[n g^{-1} \int a(\cos \theta)^{-1} d \theta\right]}{\cos ^{n+1} \theta} d \theta+C\right\} .
\end{aligned}
$$

For the case $a=0, n=2$ and the initial conditions $V=V_{0}$ and $\theta=\phi$ we obtain

$$
\begin{aligned}
V^{2} & =\frac{g}{2 K} \frac{1}{\cos ^{2} \theta[C-f(\theta)]} \\
t & =-\frac{1}{(2 g K)^{1 / 2}} \int_{\phi}^{\theta} \frac{d \theta}{\cos ^{2} \theta[C-f(\theta)]^{1 / 2}} \\
x & =-\frac{1}{2 K} \int_{\phi}^{\theta} \frac{d \theta}{\cos ^{2} \theta[C-f(\theta)]} \\
y & =-\frac{1}{2 K} \int_{\phi}^{\theta} \frac{\tan \theta d \theta}{\cos ^{2} \theta[C-f(\theta)]} \\
s & =\frac{1}{2 K} \log \frac{C-f(\theta)}{C-f(\phi)}
\end{aligned}
$$

where

and

$$
C=\frac{g}{2 K\left(V_{0} \cos \phi\right)^{2}}+f(\phi)
$$

$$
f(\theta)=\int \sec ^{3} \theta d \theta=\frac{1}{2}[\tan \theta \sec \theta+\log (\tan \theta+\sec \theta)] .
$$

5. Variational equations. ${ }^{2}$ The theory of differential variations was developed during the first world war by G. A. Bliss, F. R. Moulton and T. H. Gronwall for the purpose

2F. R. Moulton, New methods in exterior ballistics, University of Chicago Press, 1926, Ch. IV. 
of finding corrections to a trajectory due to non-standard ballistic conditions. A set of linear differential equations was derived which had to be solved in order to obtain these corrections. This was carried out by numerical methods, in a manner similar to that used in the solution of the equations of motion.

For a given set of initial conditions

$$
\begin{array}{ll}
x(0)=0, & y(0)=0, \\
x^{\prime}(0)=x_{0}^{\prime}, & y^{\prime}(0)=y_{0}^{\prime},
\end{array}
$$

Eqs. (1) have a definite solution. In terms of $t$ as independent variable, let this solution be represented by the functions

$$
\begin{array}{ll}
x=\Phi(t), & y=\Psi(t), \\
x^{\prime}=\Phi^{\prime}(t), & y^{\prime}=\Psi^{\prime}(t) .
\end{array}
$$

We now introduce two types of perturbations in the original equations (1): we introduce small perturbations in the normal forces acting upon the projectile, thus changing Eqs. (1) to the form

$$
\begin{aligned}
& \frac{d^{2} x}{d t^{2}}=-E \frac{d x}{d t}+X \\
& \frac{d^{2} y}{d t^{2}}=-E \frac{d y}{d t}+Y-g
\end{aligned}
$$

where $X$ and $Y$ are the components of acceleration due to these forces, in the $x$ and $y$ directions, respectively; at the same time we change the initial conditions given in (14) by the addition of small increments $\alpha$ and $\beta$ to the values of $x_{0}^{\prime}$ and $y_{0}^{\prime}$, respectively. The new initial conditions thus become

$$
\begin{array}{ll}
x(0)=0, & y(0)=0, \\
x^{\prime}(0)=x_{0}^{\prime}+\alpha, & y^{\prime}(0)=y_{0}^{\prime}+\beta .
\end{array}
$$

'The solution to the new set of differential equations (16) with initial conditions (17) may be written in the form

$$
\begin{array}{ll}
x=\Phi(t)+\xi(t), & y=\Psi(t)+\eta(t), \\
x^{\prime}=\Phi^{\prime}(t)+\xi^{\prime}(t), & y^{\prime}=\Psi^{\prime}(t)+\eta^{\prime}(t),
\end{array}
$$

where $\xi(t)$ and $\eta(t)$ are small compared with $\Phi(t)$ and $\Psi(t)$.

Let us now make the assumption that $E=R / V$ is a function of $x^{\prime}$ and $y^{\prime}$ but not of $y$; and that it may be expanded as a power series in terms $\xi^{\prime}=x^{\prime}-\Phi$ and $\eta^{\prime}=y^{\prime}-$ $\Psi^{\prime}$. Substituting (18) into (1) and neglecting all differentials of higher order than the first, we obtain

$$
\begin{aligned}
& \frac{d^{2} \Phi}{d t^{2}}+\frac{d^{2} \xi}{d t^{2}}=-\left[E_{0}+\frac{\partial E_{0}}{\partial x^{\prime}} \xi^{\prime}+\frac{\partial E_{0}}{\partial y^{\prime}} \eta^{\prime}\right]\left(\Phi^{\prime}+\xi^{\prime}\right)+X \\
& \frac{d^{2} \Psi}{d t^{2}}+\frac{d^{2} \eta}{d t^{2}}=-\left[E_{0}+\frac{\partial E_{0}}{\partial x^{\prime}} \xi^{\prime}+\frac{\partial E_{0}}{\partial y^{\prime}} \eta^{\prime}\right]\left(\Psi^{\prime}+\eta^{\prime}\right)+Y-g
\end{aligned}
$$


where $E_{0}$ is evaluated at $\Phi^{\prime}, \Psi^{\prime}$. Since (15) is a solution of Eqs. (1) it follows that

$$
\begin{aligned}
& \frac{d^{2} \Phi}{d t^{2}}=-E_{0} \Phi^{\prime} \\
& \frac{d^{2} \Psi}{d t^{2}}=-E_{0} \Psi^{\prime}-g .
\end{aligned}
$$

Hence, substituting into (19) and neglecting quadratic terms in $\xi^{\prime}, \eta^{\prime}$ we obtain

$$
\begin{aligned}
& \frac{d^{2} \xi}{d t^{2}}=-E_{0} \xi^{\prime}-\Phi^{\prime}\left[\frac{\partial E_{0}}{\partial x^{\prime}} \xi^{\prime}+\frac{\partial E_{0}}{\partial y^{\prime}} \eta^{\prime}\right]+X \\
& \frac{d^{2} \eta}{d t^{2}}=-E_{0} \eta^{\prime}-\Psi^{\prime}\left[\frac{\partial E_{0}}{\partial x^{\prime}} \xi^{\prime}+\frac{\partial E_{0}}{\partial y^{\prime}} \eta^{\prime}\right]+Y
\end{aligned}
$$

or,

$$
\begin{aligned}
& \frac{d^{2} \xi}{d t^{2}}=-\left(E_{0}+\Phi^{\prime} \frac{\partial E_{0}}{\partial x^{\prime}}\right) \xi^{\prime}-\Phi^{\prime} \frac{\partial E_{0}}{\partial y^{\prime}} \eta^{\prime}+X \\
& \frac{d^{2} \eta}{d t^{2}}=-\Psi^{\prime} \frac{\partial E_{0}}{\partial x^{\prime}} \xi^{\prime}-\left(E_{0}+\Psi^{\prime} \frac{\partial E_{0}}{\partial y^{\prime}}\right) \eta^{\prime}+Y .
\end{aligned}
$$

Setting $E=K G(V)$ (i.e. $H(y) \equiv 1$ ), we get

$$
\begin{aligned}
& \frac{\partial E_{0}}{\partial x^{\prime}}=K \frac{\partial G}{\partial V} \frac{\Phi^{\prime}}{V}=\frac{E_{0}}{V G} \Phi^{\prime} \frac{\partial G}{\partial V}, \\
& \frac{\partial E_{0}}{\partial y^{\prime}}=K \frac{\partial G}{\partial V} \frac{\Psi^{\prime}}{V}=\frac{E_{0}}{V G} \Psi^{\prime} \frac{\partial G}{\partial V} .
\end{aligned}
$$

Finally, substituting (23) into (22),

$$
\begin{aligned}
& \frac{d^{2} \xi}{d t^{2}}=\frac{d \xi^{\prime}}{d t}=P_{1} \xi^{\prime}+P_{2} \eta^{\prime}+X \\
& \frac{d^{2} \eta}{d t^{2}}=\frac{d \eta^{\prime}}{d t}=Q_{1} \xi^{\prime}+Q_{2} \eta^{\prime}+Y
\end{aligned}
$$

where

$$
\begin{aligned}
& P_{1}=-\left(E_{0}+\Phi^{\prime^{2}} \frac{E_{0}}{V G} \frac{\partial G}{\partial V}\right), \\
& P_{2}=Q_{1}=\Phi^{\prime} \Psi^{\prime} \frac{E_{0}}{V G} \frac{\partial G}{\partial V} \\
& Q_{2}=-\left(E_{0}+\Psi^{\prime 2} \frac{E_{0}}{V G} \frac{\partial G}{\partial V}\right) .
\end{aligned}
$$

This set of equations may also be written in the form 


$$
\begin{aligned}
& \frac{d \xi}{d t}=0+\xi^{\prime}+0+0 \\
& \frac{d \xi^{\prime}}{d t}=0+P_{1} \xi^{\prime}+0+P_{2} \eta^{\prime}+X \\
& \frac{d \eta}{d t}=0+0+0+\eta^{\prime} \\
& \frac{d \eta^{\prime}}{d t}=0+Q_{1} \xi^{\prime}+0+Q_{2} \eta^{\prime}+Y .
\end{aligned}
$$

The quantities $E_{0}, \Phi^{\prime}, \partial G / \partial V$ etc. in the expressions for $P_{1}, P_{2}, Q_{1}, Q_{2}$ are defined for the unperturbed trajectory, which we shall assume has been solved. Consequently Eqs. (24) are a set of linear differential equations, the solution to which will give the quantities $\xi, \xi^{\prime}, \eta, \eta^{\prime}$ which represent the increments in $x, x^{\prime}, y, y^{\prime}$ due to a change $\alpha, \beta$ in the initial conditions and the perturbation forces $X, Y$.

6. Solution of equations for perturbed initial conditions. We shall first obtain a solution to Eqs. (24) for the case $X=Y=0$. Under these conditions Eqs. (24) are reduced to a system of homogenous linear equations.

Let us assume that we have found two sets of solutions:

for the initial conditions

$$
\begin{aligned}
\xi^{\prime}(t) & =\xi_{1}^{\prime}(t) \\
\eta^{\prime}(t) & =\eta_{1}^{\prime}(t)
\end{aligned}
$$

$$
\begin{aligned}
& \xi^{\prime}(0)=\xi_{1}^{\prime}(0) \\
& \eta^{\prime}(0)=\eta_{1}^{\prime}(0),
\end{aligned}
$$

and

$$
\begin{aligned}
\xi^{\prime}(t) & =\xi_{2}^{\prime}(t) \\
\eta^{\prime}(t) & =\eta_{2}^{\prime}(t)
\end{aligned}
$$

for the initial conditions

$$
\begin{aligned}
\xi^{\prime}(0) & =\xi_{2}^{\prime}(0) \\
\eta^{\prime}(0) & =\eta_{2}^{\prime}(0) .
\end{aligned}
$$

It follows from the theory of linear differential equations that all solutions of Eqs. (24) may then be written in the form

$$
\begin{aligned}
& \xi^{\prime}(t)=\iota_{1} \xi_{1}^{\prime}(t)+C_{2} \xi_{2}^{\prime}(t), \\
& \eta^{\prime}(t)=C_{1} \eta_{1}^{\prime}(t)+C_{2} \eta_{2}^{\prime}(t),
\end{aligned}
$$

where $C_{1}, C_{2}$ are constants, provided the determinant

$$
D(t)=\left|\begin{array}{cc}
\xi_{1}^{\prime}(t) & \xi_{2}^{\prime}(t) \\
\eta_{1}^{\prime}(t) & \eta_{2}^{\prime}(t)
\end{array}\right| \neq 0 .
$$


It is also known that the value of this determinant is given by

$$
D(t)=D(0) \exp \left[\int_{0}^{t}\left(P_{1}+Q_{2}\right) d \dot{t}\right] .
$$

For the quadratic law of air resistance $E=K G(V)=K V$. Equations (25) then reduce to

$$
\begin{aligned}
& \frac{d \xi^{\prime}}{d t}=-K V\left(1+\frac{\Phi^{\prime 2}}{V^{2}}\right) \xi^{\prime}-\frac{K \Phi^{\prime} \Psi^{\prime}}{V} \eta^{\prime} \\
& \frac{d \eta^{\prime}}{d t}=-\frac{K \Phi^{\prime} \Psi^{\prime}}{V} \xi^{\prime}-K V\left(1+\frac{\Psi^{\prime 2}}{V^{2}}\right) \eta^{\prime} .
\end{aligned}
$$

These may also be written in the form

$$
\begin{aligned}
& \frac{d \xi^{\prime}}{d t}=-K V\left(1+\cos ^{2} \theta\right) \xi^{\prime}-K V \sin \theta \cos \theta \eta^{\prime} \\
& \frac{d \eta^{\prime}}{d t}=-K V \sin \theta \cos \theta \xi^{\prime}-K V\left(1+\sin ^{2} \theta\right) \eta^{\prime}
\end{aligned}
$$

where

$$
V=\left(\frac{g}{K}\right)^{1 / 2} \frac{1}{\cos \theta[2 C-2 f(\theta)]^{1 / 2}} \quad \text { (Eq. 9). }
$$

In order to solve Eqs. (30) it is necessary to find a set of two particular solutions to this system. Such a set of solutions is in general furnished by the derivatives of the solution to Eqs. (1) with respect to the parameters introduced by the initial values. Let the solution to Eqs. (1) be given by

$$
x^{\prime}=\Phi^{\prime}\left(t, x_{0}^{\prime}, y_{0}^{\prime}\right), \quad y^{\prime}=\Psi^{\prime}\left(t, x_{0}^{\prime}, y_{0}^{\prime}\right)
$$

then

$$
\xi^{\prime}=\frac{\partial \Phi^{\prime}}{\partial x_{0}^{\prime}}, \quad \eta^{\prime}=\frac{\partial \Psi^{\prime}}{\partial x_{0}^{\prime}} \quad \text { and } \quad \xi^{\prime}=\frac{\partial \Phi^{\prime}}{\partial y_{0}^{\prime}}, \quad \eta^{\prime}=\frac{\partial \Psi^{\prime}}{\partial y_{0}^{\prime}}
$$

satisfy Eqs. (30). Furthermore, since $t$ is not contained explicitly in Eqs. (1), it may be easily verified that

$$
\xi^{\prime}=\frac{d \Phi^{\prime}}{d t}, \quad \eta^{\prime}=\frac{d \Psi^{\prime}}{d t}
$$

is also a solution of Eqs. (30). For convenience, we choose the latter solution and also the derivatives of $\Phi^{\prime}, \Psi^{\prime}$ with respect to the parameter $C$. These are given in (31) and (32) below:

$$
\begin{aligned}
\xi_{1}^{\prime}(t) & =\frac{1}{\cos \theta[2 C-2 f(\theta)]}, \\
\eta_{1}^{\prime}(t) & =\frac{\tan \theta}{\cos \theta[2 C-2 f(\theta)]}+1,
\end{aligned}
$$


with the initial conditions

$$
\begin{aligned}
\xi_{1}^{\prime}(0) & =\frac{K V_{0}^{2} \cos \phi}{g}, \\
\eta_{1}^{\prime}(0) & =\frac{K V_{0}^{2} \sin \phi}{g}+1 ;
\end{aligned}
$$

and

$$
\begin{aligned}
& \xi_{2}^{\prime}(t)=\xi_{1}^{\prime}(t) \int_{\phi}^{\theta} \frac{d \theta}{\cos ^{2} \theta[2 C-2 f(\theta)]^{3 / 2}}-\frac{1}{[2 C-2 f(\theta)]^{3 / 2}}, \\
& \eta_{2}^{\prime}(t)=\eta_{1}^{\prime}(t) \int_{\phi}^{\theta} \frac{d \theta}{\cos ^{2} \theta[2 C-2 f(\theta)]^{3 / 2}}-\frac{\tan \theta}{[2 C-2 f(\theta)]^{3 / 2}},
\end{aligned}
$$

with the initial conditions

$$
\begin{aligned}
& \xi_{2}^{\prime}(0)=-\left(\frac{K}{g}\right)^{3 / 2}\left(V_{0} \cos \phi\right)^{3}, \\
& \eta_{2}^{\prime}(0)=-\left(\frac{K}{g}\right)^{3 / 2}\left(V_{0} \cos \phi\right)^{3} \tan \phi .
\end{aligned}
$$

We will now evaluate the determinant $D(t)$. We note that

$$
D(0)=\left|\begin{array}{ll}
\frac{1}{g} K V_{0}^{2} \cos \phi & -\left(\frac{K}{g}\right)^{3 / 2}\left(V_{0} \cos \phi\right)^{3} \\
\frac{1}{g} K V_{0}^{2} \sin \phi+1-\left(\frac{K}{g}\right)^{3 / 2}\left(V_{0} \cos \phi\right)^{3} \tan \phi
\end{array}\right|=\left(\frac{K}{g}\right)^{3 / 2}\left(V_{0} \cos \phi\right)^{3} .
$$

On the other hand

$\exp \left[\int_{0}^{t}\left(P_{1}+Q_{2}\right) d t\right]=\exp \left(-3 K \int_{0}^{t} V d t\right)=\left(\frac{g}{K}\right)^{3 / 2}\left(V_{0} \cos \phi\right)^{-3}[2 C-2 f(\theta)]^{-3 / 2}$

Hence,

$$
D(t)=D(0) \exp \left[\int_{0}^{\ell}\left(P_{1}+Q_{2}\right) d t\right]=[2 C-2 f(\theta)]^{-3 / 2} .
$$

It is apparent that $D(t)$ is not equal to zero, except when $\phi=90^{\circ}$. This case will be discussed separately in Sec. 12.

7. Solution of equations for perturbed forces. We are now ready to solve the set of non-homogeneous equations (24). We shall assume that the solution has the same form as (26) with the exception that $C_{1}, C_{2}$ are now functions of $t$ rather than constants; namely,

$$
\begin{gathered}
\xi^{\prime}(t)=C_{1}(t) \xi_{1}^{\prime}(t)+C_{2}(t) \xi_{2}^{\prime}(t), \\
\eta^{\prime}(t)=C_{1}(t) \eta_{1}^{\prime}(t)+C_{2}(t) \eta_{2}^{\prime}(t) .
\end{gathered}
$$


Differentiating (33), and remembering that (33) with $C_{1}, C_{2}$ constant is a solution to (24) when $X=Y=0$, we obtain

$$
\begin{aligned}
& \xi_{1}^{\prime}(t) \frac{d \iota_{1}}{d t}+\xi_{2}^{\prime}(t) \frac{d C_{2}}{d t}=X \\
& \eta_{1}^{\prime}(t) \frac{d C_{1}}{d t}+\eta_{2}^{\prime}(t) \frac{d C_{2}}{d t}=Y .
\end{aligned}
$$

Hence,

and

$$
\frac{d C_{1}}{d t}=D^{-1}(t)\left|\begin{array}{cc}
X & \xi_{2}^{\prime}(t) \\
Y & \eta_{2}^{\prime}(t)
\end{array}\right|=f_{1}(t)
$$

$$
\frac{d C_{2}}{d t}=D^{-1}(t)\left|\begin{array}{cc}
\xi_{1}^{\prime}(t) & X \\
\eta_{1}^{\prime}(t) & Y
\end{array}\right|=f_{2}(t)
$$

Integrating we obtain

$$
\begin{aligned}
& C_{1}(t)=B_{1}+\int_{0}^{t} f_{1}(t) d t=B_{1}+F_{1}(t) \\
& C_{2}(t)=B_{2}+\int_{0}^{t} f_{2}(t) d t=B_{2}+F_{2}(t)
\end{aligned}
$$

where $B_{1}, B_{2}$ are constants which may be determined from the initial conditions. Substituting these values into (33) we finally obtain a solution to Eqs. (24),

$$
\begin{aligned}
& \xi^{\prime}(t)=F_{1}(t) \xi_{1}^{\prime}(t)+F_{2}(t) \xi_{2}^{\prime}(t)+B_{1} \xi_{1}^{\prime}(t)+B_{2} \xi_{2}^{\prime}(t), \\
& \eta^{\prime}(t)=F_{1}(t) \eta_{1}^{\prime}(t)+F_{2}(t) \eta_{2}^{\prime}(t)+B_{1} \eta_{1}^{\prime}(t)+B_{2} \eta_{2}^{\prime}(t) .
\end{aligned}
$$

For normal initial conditions $B_{1}=B_{2}=0$, hence

$$
\begin{aligned}
& \xi^{\prime}(t)=F_{1}(t) \xi_{1}^{\prime}(t)+F_{2}(t) \xi_{2}^{\prime}(t), \\
& \eta^{\prime}(t)=F_{1}(t) \eta_{1}^{\prime}(t)+F_{2}(t) \eta_{2}^{\prime}(t) .
\end{aligned}
$$

8. Quasi-Neutonian resistance. Equations (1) express the path of a projectile under the influence of a law of air resistance $E=R / V$. If the resistance is slightly perturbed, so that only differentials of the first order must be considered, while differentials of the second or higher order may be neglected, the resistance is then given by $E+\Delta E$. When this expression is substituted into Eqs. (1), Eqs. (16) are obtained with

$$
\begin{gathered}
X=-\Delta E V \cos \theta, \\
Y=-\Delta E V \sin \theta .
\end{gathered}
$$

It follows that for the case of quasi-Newtonian resistance Eq. (35) may be written in the form 


$$
\begin{aligned}
& f_{1}(t)=-\Delta E\left|\begin{array}{rr}
\cos \theta & \xi_{2}^{\prime} \\
\sin \theta & \eta_{2}^{\prime}
\end{array}\right| V[2 C-2 f(\theta)]^{3 / 2}, \\
& f_{2}(t)=-\Delta E\left|\begin{array}{lr}
\xi_{1}^{\prime} & \cos \theta \\
\eta_{1}^{\prime} & \sin \theta
\end{array}\right| V[2 C-2 f(\theta)]^{3 / 2} .
\end{aligned}
$$

Consequently,

$$
\begin{aligned}
& F_{1}=\int_{\phi}^{\theta} \frac{\Delta E}{K}\left|\begin{array}{cc}
\cos \theta & \xi_{2}^{\prime} \\
\sin \theta & \eta_{2}^{\prime}
\end{array}\right| \frac{[2 C-2 f(\theta)]^{1 / 2}}{\cos ^{3} \theta} d \theta, \\
& F_{2}=\int_{\phi}^{\theta} \frac{\Delta E}{K}\left|\begin{array}{cc}
\xi_{1}^{\prime} & \cos \theta \\
\eta_{1}^{\prime} & \sin \theta
\end{array}\right| \frac{[2 C-2 f(\theta)]^{1 / 2}}{\cos ^{3} \theta} d \theta .
\end{aligned}
$$

We now may write expressions for the increments

$$
\begin{aligned}
\xi & =-\int_{\phi}^{\theta} \frac{\left(F_{1} \xi_{1}^{\prime}+F_{2} \xi_{2}^{\prime}\right) d \theta}{(g K)^{1 / 2} \cos ^{2} \theta[2 C-2 f(\theta)]^{1 / 2}} \\
\xi^{\prime} & =\frac{d \xi}{d t}=F_{1} \xi_{1}^{\prime}+F_{2} \xi_{2}^{\prime} \\
\eta & =-\int_{\phi}^{\theta} \frac{\left(F_{1} \eta_{1}^{\prime}+F_{2} \eta_{2}^{\prime}\right) d \theta}{(g K)^{1 / 2} \cos ^{2} \theta[2 C-2 f(\theta)]^{1 / 2}} \\
\eta^{\prime} & =\frac{d \eta}{d t}=F_{1} \eta_{1}^{\prime}+F_{2} \eta_{2}^{\prime} .
\end{aligned}
$$

Equations (10), (11), (12), and (41) thus give the complete solution for a trajectory with quasi-Newtonian resistance. The procedure for carrying out a solution consists of several steps. First we solve Eqs. (10), (11), and (12), using a value of $K$ that would give as closely as possible a true estimate of the resistance function. After this has been completed the function $\Delta E=E-K V$ is computed. $(E=K G(V) H(y)$ is the true resistance function, and may in practice also include the variation in density due to altitude.) Then the corrections $\xi, \xi^{\prime}, \eta, \eta^{\prime}$ due to a change $\Delta E$ in the resistance function are computed from Eqs. (41). The true values of $t, x, x^{\prime}, y, y^{\prime}$ which we shall denote by $\bar{t}, \bar{x}, \bar{x}^{\prime}, \bar{y}, \bar{y}^{\prime}$ are then given by the relations

$$
\begin{aligned}
& \bar{t}=t \\
& \bar{x}=x+\xi \\
& \bar{x}^{\prime}=x^{\prime}+\xi^{\prime} \\
& \bar{y}_{: .}=y+\eta \\
& \bar{y}^{\prime}=y^{\prime}+\eta^{\prime} .
\end{aligned}
$$


In order to test the applicability of this method to practical ballistic problems a portion of a trajectory based on the standard Army resistance function $G_{2}$ was calculated by the usual method of successive approximations. The initial values were taken as follows:

Initial velocity $=300 \mathrm{~m} / \mathrm{s}_{\text {r }}$

Ballistic coefficient $=1$,

Angle of elevation $=60^{\circ}$.

The solution obtained is compared in the table below with the solution by the method proposed in this paper. There seems to be perfect agreement between the two results, as far as the computation was carried.

\begin{tabular}{ccc|cr}
\multicolumn{3}{c|}{ Successive Approximations } & \multicolumn{2}{c}{ New Method } \\
\hline $\begin{array}{c}t \\
\text { sec. }\end{array}$ & $\begin{array}{c}\bar{x} \\
\text { meters }\end{array}$ & $\begin{array}{c}\bar{y} \\
\text { meters }\end{array}$ & $\begin{array}{c}\bar{x} \\
\text { meters }\end{array}$ & $\begin{array}{c}\bar{y} \\
\text { meters }\end{array}$ \\
\hline 0.00 & 0.0 & 0.0 & 0.0 & 0.0 \\
1.00 & 146.8 & 249.5 & 146.8 & 249.5 \\
2.00 & 287.9 & 479.6 & 287.8 & 479.5 \\
3.00 & 424.0 & 692.0 & 424.0 & 691.9 \\
4.00 & 555.8 & 888.0 & 555.8 & 887.9 \\
5.00 & 683.8 & 1068.5 & 683.7 & 1068.4 \\
6.00 & 808.2 & 1234.5 & 808.1 & 1234.4 \\
7.00 & 929.6 & 1386.7 & 929.5 & 1386.6 \\
8.00 & 1048.1 & 1525.7 & 1048.0 & 1525.5 \\
\hline
\end{tabular}

9. The adjoint system of equations. In many ballistic problems only the terminal conditions of the trajectory, such as the range of the projectile when it strikes the ground, are required. In that event it is not necessary to obtain the path of the complete trajectory. By solving the system of equations adjoint to (25) it is possible to obtain directly the terminal values and thus to eliminate a large percentage of the computational work. In addition to this gain the method of the adjoint system yields expressions that can be used to find the effects upon the normal trajectory due to non-standard conditions of the air, such as wind or temperature variations. There is a third advantage to the computer in this method in that the final results are given for equal intervals of $\theta$, which is not true in the case of the formulas derived in the preceding section.

The set of equations

$$
\begin{aligned}
& -\frac{d \rho}{d t}=0+0+0+0 \\
& -\frac{d \rho^{\prime}}{d t}=\rho+P_{1} \rho^{\prime}+0+Q_{1} \sigma^{\prime} \\
& -\frac{d \sigma}{d t}=0+0+0+0 \\
& -\frac{d \sigma^{\prime}}{d t}=0+P_{2} \rho^{\prime}+\sigma+Q_{2} \sigma^{\prime}
\end{aligned}
$$


whose coefficients are the same as those of Eqs. (25) with rows and columns interchanged, is known as the system of equations adjoint to (25). If a solution to (43) is found for the variables $\rho, \rho^{\prime}, \sigma, \sigma^{\prime}$ the quantities $\xi, \xi^{\prime}, \eta, \eta^{\prime}$ may then be expressed in terms of these variables. The relationship between these two sets of variables may be easily derived.

Multiplying Eqs. (25) by $\rho, \rho^{\prime}, \sigma, \sigma^{\prime}$ respectively, and adding we obtain

$$
\begin{aligned}
\rho \frac{d \xi}{d t} & +\rho^{\prime} \frac{d \xi^{\prime}}{d t}+\sigma \frac{d \eta}{d t}+\sigma^{\prime} \frac{d \eta^{\prime}}{d t} \\
& =\xi^{\prime}\left(\rho+P_{1} \rho^{\prime}+Q_{1} \sigma^{\prime}\right)+\eta^{\prime}\left(\sigma+P_{2} \rho^{\prime}+Q_{2} \sigma^{\prime}\right)+\left(\rho^{\prime} X+\sigma^{\prime} Y\right) .
\end{aligned}
$$

Substituting Eqs. (43) we obtain

$$
\rho \frac{d \xi}{d t}+\rho^{\prime} \frac{d \xi^{\prime}}{d t}+\sigma \frac{d \eta}{d t}+\sigma^{\prime} \frac{d \eta^{\prime}}{d t}+\xi \frac{d \rho}{d t}+\xi^{\prime} \frac{d \rho^{\prime}}{d t}+\eta \frac{d \sigma}{d t}+\eta^{\prime} \frac{d \sigma^{\prime}}{d t}=\rho^{\prime} X+\sigma^{\prime} Y
$$

or

$$
\frac{d}{d t}\left(\rho \xi+\rho^{\prime} \xi^{\prime}+\sigma \eta+\sigma^{\prime} \eta^{\prime}\right)=\rho^{\prime} X+\sigma^{\prime} Y
$$

Finally,

$$
\left[\rho \xi+\rho^{\prime} \xi^{\prime}+\sigma \eta+\sigma^{\prime} \eta^{\prime}\right]_{0}^{T}=\int_{0}^{T}\left(\rho^{\prime} X+\sigma^{\prime} Y\right) d t
$$

where $T$ is the time at the terminal point of the unperturbed or Newtonian trajectory.

10. Solution of the Adjoint System. It follows immediately from the first and third of Eqs. (43) that $\rho=$ constant and that $\sigma=$ constant. We shall denote these constants by $\bar{\rho}$ and $\bar{\sigma}$, respectively. The second and fourth equations may then be written

$$
\begin{aligned}
& -\frac{d \rho^{\prime}}{d t}=\bar{\rho}+P_{1} \rho^{\prime}+Q_{1} \sigma^{\prime} \\
& -\frac{d \sigma^{\prime}}{d t}=\bar{\sigma}+P_{2} \rho^{\prime}+Q_{2} \sigma^{\prime},
\end{aligned}
$$

or

$$
\begin{aligned}
& -\frac{d \rho^{\prime}}{d t}=\bar{\rho}-\left(E_{0}+\Phi^{\prime 2} \frac{E_{0}}{V G} \frac{\partial G}{\partial V}\right) \rho^{\prime}-\Phi^{\prime} \Psi^{\prime} \frac{E_{0}}{V G} \frac{\partial G}{\partial V} \sigma^{\prime} \\
& -\frac{d \sigma^{\prime}}{d t}=\bar{\sigma}-\Phi^{\prime} \Psi^{\prime} \frac{E_{0}}{V G} \frac{\partial G}{\partial V} \rho^{\prime}-\left(E_{0}+\Psi^{\prime 2} \frac{E_{0}}{V G} \frac{\partial G}{\partial V}\right) \sigma^{\prime} .
\end{aligned}
$$

It may be verified that one solution of this system is

$$
\bar{\rho}_{1} \Phi^{\prime}+\bar{\sigma}_{1} \Psi^{\prime}+\rho_{1}^{\prime} \Phi^{\prime \prime}+\sigma_{1}^{\prime} \Psi^{\prime \prime}=-K_{1} \quad \text { (constant). }
$$

This follows from the fact that $\xi=\Phi^{\prime}, \xi^{\prime}=\Phi^{\prime \prime}, \eta=\Psi^{\prime}, \eta^{\prime}=\Psi^{\prime \prime}$ is a solution to Eqs. (24), and from (46) which reduces to

$$
\frac{d}{d t}\left(\rho \xi+\rho^{\prime} \xi^{\prime}+\sigma \eta+\sigma^{\prime} \eta^{\prime}\right)=0 \quad \text { for } \quad X=0, \quad Y=0 .
$$


Transposing the terms of Eq. (50) and dividing by $\Phi^{\prime \prime}$ we obtain

$$
-\rho_{1}^{\prime}=\frac{\bar{\rho}_{1} \Phi^{\prime}+\bar{\sigma}_{1} \Psi^{\prime}+\sigma_{1}^{\prime} \Psi^{\prime \prime}+K_{1}}{\Phi^{\prime \prime}}
$$

which may be used with the second equation of (49) to eliminate $\rho_{1}^{\prime}$; thus

$$
\begin{aligned}
-\frac{d \sigma_{1}^{\prime}}{d t}=\bar{\sigma}_{1} & +\Phi^{\prime} \Psi^{\prime} \frac{E_{0}}{V G} \frac{\partial G}{\partial V} \frac{\bar{\rho}_{1} \Phi^{\prime}+\bar{\sigma}_{1} \Psi^{\prime}+\sigma_{1}^{\prime} \Psi^{\prime \prime}+K_{1}}{\Phi^{\prime \prime}} \\
& -\left(E_{0}+\Psi^{\prime} \frac{E_{0}}{V G} \frac{\partial G}{\partial V}\right) \sigma_{1}^{\prime} .
\end{aligned}
$$

Remembering that $\Phi^{\prime}=x^{\prime}, \Psi^{\prime}=y^{\prime}$ of the undisturbed or Newtonian trajectory, and that $\Phi^{\prime \prime}=-E_{0} \Phi^{\prime}$, the preceding equation reduces to a linear differential equation of the first order which may be solved immediately; namely

$$
\frac{d \sigma_{1}^{\prime}}{d t}+A(t) \sigma_{1}^{\prime}+B(t)=0
$$

where

$$
\begin{aligned}
& A(t)=g \Psi^{\prime} \frac{d \log G}{V d V}-E_{0}, \\
& B(t)=\bar{\sigma}_{1}-\Psi^{\prime} \frac{d \log G}{V d V}\left(\bar{\rho}_{1} \Phi^{\prime}+\bar{\sigma}_{1} \Psi^{\prime}+K_{1}\right) .
\end{aligned}
$$

The solution of this equation is

$$
\sigma_{1}^{\prime}=\exp \left(-\int_{0}^{t} A(t) d t\right)\left[C_{3}-\int_{0}^{t} B(t) \exp \left(\int_{0}^{t} A(t) d t\right) d t\right] .
$$

The expression for $\rho_{1}^{\prime}$ is given by Eq. (51) above.

We shall want only the solution for the case $\sigma_{1}^{\prime}=0$ at $t=T$. Hence Eq. (54) may be written

$$
\begin{aligned}
\sigma_{1}^{\prime}(t) & =\exp \left(-\int_{0}^{t} A(t) d t\right) \\
& \cdot\left[\int_{0}^{T} B(t) \exp \left(\int_{0}^{t} A(t) d t\right) d t-\int_{0}^{t} B(t) \exp \left(\int_{0}^{t} A(t) d t\right) d t\right] .
\end{aligned}
$$

We are particularly interested in the solution of the above equation for the special case $E=K G(V)=K V$; hence $d \log G / V d V=1 / V^{2}$. From Eqs. (3), (4), (5) and (9) it follows that,

$$
A(t)=\frac{g \sin \theta-K V^{2}}{V}
$$

and

$$
\int_{0}^{t} A(t) d t=-\int_{\phi}^{\theta} \frac{g \sin \theta-K V^{2}}{g \cos \theta} d \theta=-\int_{\phi}^{\theta} \tan \theta d \theta+\frac{1}{2} \int_{\phi}^{\theta} \frac{d \theta}{\cos ^{3} \theta[C-f(\theta)]} .
$$


This may be integrated, giving the solution

$$
\int_{0}^{t} A(t) d t=\log \left\{\frac{\cos \theta}{\cos \phi}\left[\frac{C-f(\phi)}{C-f(\theta)}\right]^{1 / 2}\right\}
$$

and finally,

$$
\exp \left(\int_{0}^{t} A(t) d t\right)=\frac{\cos \theta}{\cos \phi}\left[\frac{C-f(\phi)}{C-f(\theta)}\right]^{1 / 2}=\left(\frac{g}{K}\right)^{1 / 2} \frac{\cos \theta}{V_{0} \cos ^{2} \phi[2 C-2 f(\theta)]^{1 / 2}} .
$$

Also,

$$
\begin{aligned}
B(t) & =\bar{\sigma}_{1}-\frac{\sin \theta}{V}\left(\bar{\rho}_{1} V \cos \theta+\bar{\sigma}_{1} V \sin \theta+K_{1}\right) \\
& =\bar{\sigma}_{1} \cos ^{2} \theta-\bar{\rho}_{1} \cos \theta \sin \theta-\frac{K_{1} \sin \theta}{V}
\end{aligned}
$$

Hence,

$$
\begin{gathered}
\int_{0}^{t} B(t) \exp \left(\int_{0}^{t} A(t) d t\right) d t=\frac{1}{K V_{0} \cos ^{2} \phi} \int_{\phi}^{\theta}\left[\left(\frac{K}{g}\right)^{1 / 2} \frac{K_{1} \sin \theta}{[2 C-2 f(\theta)]^{1 / 2}}\right. \\
\left.-\frac{\bar{\sigma}_{1} \cos \theta-\bar{\rho}_{1} \cos \theta}{2 C-2 f(\theta)}\right] d \theta
\end{gathered}
$$

Finally,

$$
\begin{aligned}
& \sigma_{1}^{\prime}=\frac{1}{(K g)^{1 / 2}} \frac{[2 C-2 f(\theta)]^{1 / 2}}{\cos \theta} \int_{\theta}^{\omega}\left[\left(\frac{K}{g}\right)^{1 / 2} \frac{K_{1} \sin \theta}{[2 C-2 f(\theta)]^{1 / 2}}-\frac{\bar{\sigma}_{1} \cos \theta-\bar{\rho}_{1} \cos \theta}{2 C-2 f(\theta)}\right] d \theta \\
& \rho_{1}^{\prime}=-\frac{\bar{\rho}_{1} \Phi^{\prime}+\bar{\sigma}_{1} \Psi^{\prime}+\sigma_{1}^{\prime} \Psi^{\prime \prime}+K_{1}}{\Phi^{\prime \prime}}
\end{aligned}
$$

where $\omega$ is the angle at the terminal point of the trajectory.

11. Range and time of flight. As is well-known, the solution derived for the adjoint system of differential equations (43) may be used to obtain the range, the total time of flight or other terminal values of the perturbed trajectory. The quantities that are of greatest interest are the range (the horizontal distance from the muzzle of the gun to the point where the projectile strikes the ground) and the time of flight.

Let us suppose that Eqs. (10), (11), and (12) have been solved for a trajectory with given initial conditions. Let $T$ be the time of flight to the ground, and $X_{T}$ the range. These equations are based on the Newtonian resistance law, and the resulting trajectory is an approximation to the true trajectory. Since the true trajectory which obeys a quasi-Newtonian resistance law is not much different from the Newtonian trajectory we shall consider only differentials of the first order. In Fig. 1, $X_{T}$ is the range of the Newtonian trajectory, $X_{T}$, is the range of the quasi-Newtonian trajectory, $P$ is the location of the projectile at time $T$, and $P 0$ is a line normal to the $x$-axis. The change in horizontal range $\xi(T)=X_{T} 0$; while $X_{T}, 0=-x^{\prime}(T) \eta(T) / y^{\prime}(T)$. Hence $X_{T} X_{T}$, the total increment in range, is given by the quantity $\xi(T)-x^{\prime}(T) \eta(T) / y^{\prime}(T)$. Likewise, the increment in time of flight, which is the time the projectile moves from $\mathrm{P}$ to $X_{T^{\prime}}$, is given by $-\eta(T) / y^{\prime}(T)$. 
In the preceding section we have found the solution to the adjoint system of differential equations. We may now assign initial conditions to $\rho, \rho^{\prime}, \sigma, \sigma^{\prime}$. Let us first assign the set of initial conditions

$$
\rho(T)=1, \quad \rho^{\prime}(T)=0, \quad \sigma(T)=\frac{x^{\prime}(T)}{y^{\prime}(T)}, \quad \sigma^{\prime}(T)=0 .
$$

Substituting these in Eq. (47) we obtain, since $\xi(0)=\eta(0)=0$

$$
\xi(T)-\frac{x^{\prime}(T)}{y^{\prime}(T)} \eta(T)=\rho^{\prime}(0) \xi^{\prime}(0)+\sigma^{\prime}(0) \eta^{\prime}(0)+\int_{0}^{T}\left(\rho^{\prime} X^{\top}+\sigma^{\prime} \sigma_{-}^{\prime} d t .\right.
$$

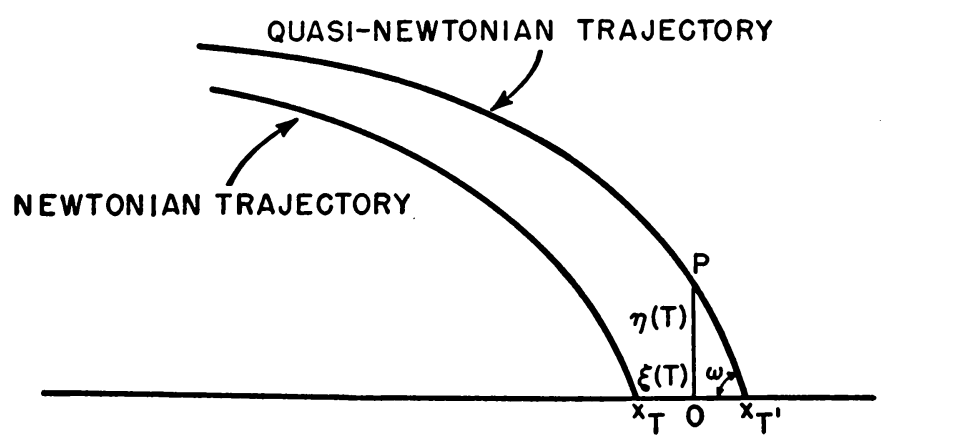

Fig. 1

It will be noticed that the left-hand side of Eq. (59) is the increment in range derived from geometrical considerations. Thus Eq. (59) gives an expression for the difference in range between Newtonian and quasi-Newtonian resistance in terms of the functions $\sigma^{\prime}$ and $\rho^{\prime}$ of the adjoint system. These are given by Eqs. (57) above.

In order to obtain the increment in time of flight we assign the initial conditions

$$
\rho(T)=0, \quad \rho^{\prime}(T)=0, \quad \sigma(T)=\frac{1}{y^{\prime}(T)}, \quad \sigma^{\prime}(T)=0 .
$$

These give, by substitution in (47)

$$
-\frac{\eta(T)}{y^{\prime}(T)}=\rho^{\prime}(0) \xi^{\prime}(0)+\sigma^{\prime}(0) \eta^{\prime}(0)+\int_{0}^{T}\left(\rho^{\prime} X+\sigma^{\prime} Y\right) d t
$$

which is an expression for the increment in time. The functions $\rho^{\prime}$ and $\sigma^{\prime}$ may again be obtained from Eqs. (57). It will be noted that the value of $K_{1}$ is not the same for range and time of flight but must be determined from Eq. (50) before solving Eqs. (57). For the increment in range $K_{1}$ is equal to zero, while for the increment in time of flight $K_{1}=1$.

A complete trajectory with the initial conditions used for the illustrative problem in Sec. 8 was computed by the standard method and by the method of the adjoint system. The ranges obtained by the two methods were identical to the nearest meter, each giving a range of $\mathbf{4 2 4 0}$ meters. The actual values obtained were $\mathbf{4 2 3 9} .8$ meters and 4240.2 meters. This may be considered complete agreement since the inaccuracy due 
to the method of successive approximations is of the same magnitude as the discrepancy. For practical ballistic problems this is far greater accuracy than is usually desired.

12. Vertical trajectories. It was pointed out in Sec. 6 that the equations derived there for a trajectory with perturbed initial conditions do not hold for a vertical trajectory, since for such a trajectory $d x / d t=0$. In this section we will derive simplified formulas for this special case. It will be of interest to note here that for the case of a vertical trajectory the solution of the equations of motion for Newtonian resistance with variation in air resistance due to an exponential law of density aloft is known. The solution may be given in terms of the function $E i(z)=\int_{-\infty}^{z}\left(e^{z} / z\right) d z$.

In one dimension the equation of motion is

$$
\frac{d^{2} y}{d t^{2}}=-E \frac{d y}{d t}-g \text {. }
$$

Let the solution to this equation for the initial conditions

$$
\begin{gathered}
y(0)=0 \\
y^{\prime}(0)=y_{0}^{\prime}
\end{gathered}
$$

be given by

$$
\begin{aligned}
y & =\Psi(t) \\
y^{\prime} & =\Psi^{\prime}(t) .
\end{aligned}
$$

Also, let the solution for non-standard initial conditions

$$
\begin{gathered}
y(0)=0 \\
y^{\prime}(0)=y_{0}^{\prime}+\beta
\end{gathered}
$$

be given by

$$
\begin{aligned}
y & =\Psi(t)+\eta(t) \\
y^{\prime} & =\Psi(t)+\eta^{\prime}(t) .
\end{aligned}
$$

If we assume in addition that $E=R / V$ is a function of $y^{\prime}=V$ alone and may be expanded in terms of $\eta^{\prime}=\left(y^{\prime}-\Psi^{\prime}\right)$, we may write

$$
\frac{d^{2} y}{d t^{2}}+\frac{d^{2} \eta^{2}}{d t^{2}}=-\left[E_{0}+\frac{d E_{0}}{d y^{\prime}} \eta^{\prime}\right]\left(\Psi^{\prime}+\eta^{\prime}\right)-g .
$$

Substituting Eq. (62) into (63) and neglecting terms in $\eta^{\prime \prime}$ we obtain

$$
\frac{d^{2} \eta}{d t^{2}}=-E_{0} \eta^{\prime}-y^{\prime} \frac{d E_{0}}{d y^{\prime}} \eta^{\prime}
$$

Since $y^{\prime}=V$ and $E$ can be written $E=K G(V)$, the above equation reduces to

$$
\frac{d^{2} \eta}{d t^{2}}=\frac{d \eta^{\prime}}{d t}=-\left[K G(V)+K V \frac{d G}{d V}\right] \eta^{\prime} .
$$


Hence

$$
\eta^{\prime}=C_{4} \exp \left[-\int\left(K G(V)+K V \frac{d G}{d V}\right) d t\right]
$$

For a small abnormal force $Y$ acting on the projectile, Eq. (62) may be written

$$
\frac{d^{2} y}{d t^{2}}=-E \frac{d y}{d t}+Y-g
$$

For this case

$$
\frac{d^{2} \eta}{d t^{2}}=\frac{d \eta^{\prime}}{d t}=-\left[K G(V)+K V \frac{d G}{d V}\right] \eta^{\prime}+Y
$$

which is a linear differential equation. The solution is given by

$$
\begin{aligned}
& \eta^{\prime}=\exp {\left[-K \int\left(G(V)+V \frac{d G}{d V}\right) d t\right] } \\
& \cdot\left\{C_{5}+\int Y \exp \left[K \int\left(G(V)+V \frac{d G}{d V}\right) d t\right] d t\right\} .
\end{aligned}
$$

For the special case $G(V)=V$

$$
\eta^{\prime}=\exp (-2 K y)\left[C_{5}+\int Y \exp (2 K y) d t\right] .
$$

